\title{
VARIOUS TYPES OF NORM-DETERMINING MANIFOLDS
}

\section{B. E. WILDER}

1. Introduction. The objectives of this paper are to prove some theorems, reminiscent of those obtained by Dixmier [3] and Ruston [4], concerning some norm-determining manifolds, and to introduce a family of Banach spaces, called spaces with property $D$, and show that this property is a generalization of some well-known conditions; for example, reflexiveness. Absolutely total hyperplanes are characterized as those whose generating functional is orthogonal to the space's canonical image, and $d$-manifolds are characterized in terms of the types of norms definable on them. Some special nonreflexive spaces, in particular $l_{1}$, are shown to have property D. Any subsequent reference to Dixmier or Ruston will refer to [3] or [4] respectively.

This paper is based on a thesis submitted in partial fulfillment of the requirements for the $\mathrm{Ph}$. D. degree at Auburn University. I would like to thank Professor J. R. Calder for the training and encouragement he has given me.

2. Preliminary definitions. The term "linear space" refers to a nondegenerate linear space over the field of real numbers. If $S$ is a linear space, then $S^{+}$denotes the set of all linear functionals on $S$, and $N(S)$ denotes the origin in $S$; if $S$ is normed, then $U(S)$ denotes the set of points in $S$ with norm 1 and $J$ denotes the canonical map of $S$ in to its second conjugate space, $S^{* *}$.

Suppose that $S$ is a linear space, that $K$ is a linear manifold in $S^{+}$, and that $h$ is a norm on $K$. Then if $x$ is in $S, A(x, K, h)=\{\mid f(x) \| f$ is in $K$ and $h(f) \leqq 1\}$. If for each $x$ in $S, A(x, K, h)$ is bounded, then $g(\cdot, K, h)$ denotes the transformation from $S$ into $E_{1}$ such that if $x$ is in $S$, then $g(x, K, h)=1$.u.b. $A(x, K, h)$. If $S$ is normed, $K$ is contained in $S^{*}$ and $h$ is the usual norm on $S^{*}$, then $A(x, K, h)$ is denoted by $A(x, K)$ and $g(\cdot, K, h)$ is denoted by $g(\cdot, K)$.

3. Some properties of absolutely total manifolds. In this section we suppose that $S$ is a normed linear space.

Definition 3.1. Suppose that $K$ is a linear manifold in $S^{*}$. The statement that $K$ is absolutely total means that if $x$ is in $S$, then $g(x, K)=\|x\|$. (An absolutely total manifold is one which is of characteristic 1 in the sense of Dixmier.)

Received by the editors August 24, 1966. 
Lemma 3.1. Suppose that $K$ is a dense linear manifold in $S^{*}$. Then $K$ is absolutely total.

Proof. Suppose that $x$ is in $S$. There is a point $f$ of $S^{*}$ such that $|f| \leqq 1$ and $|f(x)|=\|x\|$. Since $K$ is dense in $S^{*}$, there is a sequence $f_{1}, f_{2}, \ldots$ each term of which is a point of $K$ with norm $\leqq 1$ and which converges to $f$ in $S^{*}$. Then $\left|f_{1}(x)\right|,\left|f_{2}(x)\right|, \cdots$ converges to $|f(x)|=\|x\|$, and $\left|f_{n}(x)\right|$ belongs to $A(x, K)$ for each $n$, so $\|x\|$ is a point or a limit point of $A(x, K)$. Since $\|x\|$ is an upper bound of $A(x, K),\|x\|=1$.u.b. $A(x, K)=g(x, K)$. Thus $K$ is absolutely total.

Ruston proved that if $S$ is complete, it is reflexive if and only if $S^{*}$ contains no proper closed total linear manifold. Using this with Lemma 3.1 we get a more complete theorem.

Theorem 3.1. If $S$ is complete, the following two statements are equivalent:

(1) $S$ is reflexive

(2) if $K$ is a linear manifold in $S^{*}$, then the following three statements are equivalent:

(a) $K$ is total,

(b) $K$ is absolutely total,

(c) $K$ is dense in $S^{*}$.

Proof. Suppose that $S$ is reflexive, and $K$ is a linear manifold in $S^{*}$. If $K$ is total, $\bar{K}$ is total and closed, so $\bar{K}=S^{*}$ and $K$ is dense in $S^{*}$. If $K$ is dense in $S^{*}$ it is absolutely total by Lemma 3.1. Clearly, any absolutely total manifold is total. Thus statement (2) is true. Conversely, if statement (2) is true, $S^{*}$ contains no proper closed total manifold and $S$ is reflexive.

Part (a) of statement (2) cannot be omitted in Theorem 3.1 since Ruston has shown that each absolutely total manifold in $c_{0}{ }^{*}$ is dense in $c_{0}{ }^{*}$. However, it can be omitted in the case of second conjugate spaces.

THEOREM 3.2. If $S$ is complete, the following two statements are equivalent:

(1) $S$ is reflexive;

(2) if $K$ is an absolutely total linear manifold in $S^{* *}$, then $K$ is dense in $S^{* *}$.

Proof. Suppose that statement (2) is true. Dixmier showed that $J(S)$ is absolutely total in $S^{* *}$, so it is dense in $S^{* *}$. Since $S$ is complete, $J(S)$ is closed in $S^{* *}$. Hence $J(S)=S^{* *}$ and $S$ is reflexive. Conversely, if $S$ is reflexive, $S^{*}$ is reflexive and each absolutely total manifold in $S^{* *}$ is dense in $S^{* *}$ by Theorem 3.1 . 
The final theorem of this section characterizes the points of $S^{* *}$ whose zero hyperplane is an absolutely total manifold in $S^{*}$. Since the next lemma is given as an exercise in [5, p. 105], it is stated here without proof.

Lemma 3.2. If $K$ is a linear manifold in $S^{*}$ and $x$ is in $S$, then $g(x, K)=d\left(J_{x}, K^{0}\right)$, where $K^{0}$ is the set of points in $S^{* *}$ which are zero on $K$.

Definition 3.2. Suppose that each of $x$ and $y$ is in $S$ and that $V$ is a subset of $S$. The statement that $y$ is orthogonal to $x$ means that if $k$ is a number, then $\|x\| \leqq\|x-k y\|$. The statement that $y$ is orthogonal to $V$ means that $y$ is orthogonal to each point of $V$.

Theorem 3.3. Suppose that $S$ is complete, that $F$ is a point of $S^{* *}$ not in $J(S)$, and that $K$ is the zero hyperplane of $F$. Then the following two statements are equivalent:

(1) $K$ is absolutely total;

(2) $F$ is orthogonal to $J(S)$.

Proof. Suppose that $K$ is absolutely total. Suppose moreover that $J_{x}$ is a point of $J(S)$ and that $k$ is a number. Since $\|x\|=g(x, K)$, $\left|J_{x}\right|=d\left(J_{x}, K^{0}\right)$ by Lemma 3.2. But $k F$ is in $K^{0}$, so $\left|J_{x}\right| \leqq\left|J_{x}-k F\right|$, and $F$ is orthogonal to $J_{x}$. Hence $F$ is orthogonal to $J(S)$. Conversely, suppose that $F$ is orthogonal to $J(S)$ but that $K$ is not absolutely total. Then there is a point $x$ of $S$ such that $g(x, K)<\|x\|$. Then $d\left(J_{x}, K^{0}\right)$ is less than $\left|J_{x}\right|$, and there is a point $G$ of $K^{0}$ such that $\left|J_{x}-G\right|<\left|J_{x}\right|$. But $K$ is a hyperplane and each of $F$ and $G$ is zero on $K$, so $G$ is a multiple of $F$, say $G=k F$. Then $\left|J_{x}-k F\right|<\left|J_{x}\right|$ and $F$ is not orthogonal to $J_{x}$, resulting in a contradiction. The statements are thus equivalent.

4. Some properties of $d$-manifolds. In this section $S$ denotes a linear space.

DEFINITION 4.1. If $S$ is normed, and $K$ is a linear manifold in $S^{*}$, the statement that $K$ is a $d$-manifold means that if $x$ is in $S$, there is a point $f$ of $K$ such that $|f|=1$ and $f(x)=\|x\|$.

Any $d$-manifold is absolutely total. To obtain an absolutely total manifold which is not a $d$-manifold, consider the set $B=\left\{b_{1}, b_{2}, \cdots\right\}$ of points in $H^{*}\left(H=\right.$ real Hilbert space) where $b_{n}(x)=x_{n}$ for each point $x=\left(x_{1}, x_{2}, \cdots\right)$ of $H$, and let $K$ denote the linear span of $B$. $K$ is absolutely total since it is dense in $H^{*}$ but there is no point of $K$ with norm one which maps the point $x$ of $H$ such that $x_{n}=1 /\left(2^{n}\right)^{1 / 2}$ onto its norm.

We now give a general characterization of $d$-manifolds. 
THEOREM 4.1. If $K$ is a total linear manifold in $S^{+}$, then the following two statements are equivalent:

(1) there exists a norm $g$ on $S$ such that $K$ is a d-manifold in $(S, g)^{*}$;

(2) there exists a norm $h$ on $K$ such that if $x$ is in $S$, then $A(x, K, h)$ is closed and bounded.

Proof. If statement (1) is true, we may take $h$ to be the usual norm in $(S, g) *$ to obtain statement (2). Suppose that statement (2) is true. Let $g$ denote the transformation $g(\cdot, K, h)$. Since $K$ is total, $g(x)>0$ if $x$ is not $N(S)$. Clearly $g(N(S))=0$. Using the definition of $g$, it is a straightforward argument to show that $g$ is a seminorm on $S$. Hence $g$ is a norm on $S$. If $f$ is a point of $K$ different from $N\left(S^{+}\right), x$ is in $S$, and $f^{\prime}=[1 / h(f)] f$, then

$$
|f(x)|=h(f)\left|f^{\prime}(x)\right| \leqq h(f) g(x) .
$$

Thus $f$ is bounded relative to $g$ and $|f| \leqq h(f)$. Hence $K$ is contained in $(S, g)^{*}$. Suppose $x$ is in $S$. Since $A(x, K, h)$ is closed, it contains its least upper bound, $g(x)$. Then there is a point $f$ of $K$ such that $h(f) \leqq 1$ and $|f(x)|=g(x)$. Then $|f| \leqq 1$ and $|f(x)|=g(x)$. Hence $K$ is a $d$ manifold in $(S, g)$.

5. Property D. In this section, $S$ denotes a nondegenerate Banach space.

Definition 5.1. The statement that $S$ has property $\mathrm{D}$ means that if $K$ is a $d$-manifold in $S^{*}$, then $K$ is dense in $S^{*}$.

Definition 5.2. If $x$ is in $S$, the statement that $x$ is a smooth point means that there is only one point $f$ of $S^{*}$ (called the unique extension of $x$ ) such that $|f|=1$ and $f(x)=\|x\|$. The statement that $S$ is smooth means that each point of $U(S)$ is a smooth point.

Theorem 5.1. Suppose that $S$ has a smooth point and that $E$ denotes the set of unique extensions of smooth points in $U(S)$. If the linear span of $E$ (denoted by $\mathrm{L}(E)$ ) is dense in $S^{*}$, then $S$ has property $\mathrm{D}$.

Proof. Suppose that $K$ is a $d$-manifold in $S^{*}$. Let $f$ be a point of $E$. Then $f$ is the unique extension of a smooth point $x$. Since $K$ is a $d$-manifold, there is a point $g$ of $K$ with norm one such that $g(x)=\|x\|$. Then $g$ is $f$ since $x$ is a smooth point. Then $E$ is contained in $K$, so $\mathrm{L}(E)$ is contained in $K$ and $K$ is dense in $S^{*}$. Hence $S$ has property D.

\section{Corollary 5.1. If $S$ is smooth, then $S$ has property D.}

Proof. The set $E$ of Theorem 5.1 contains all the points of $U\left(S^{*}\right)$ which attain their norm, so $\mathrm{L}(E)$ contains all the points of $S^{*}$ which attain their norm. Bishop and Phelps proved [1] that this set is dense in $S^{*}$, so $\mathrm{L}(E)$ is dense in $S^{*}$ and $S$ has property $\mathrm{D}$. 
Corollary 5.2. If $S^{*}$ is strictly convex, then $S$ has property D.

Since each absolutely total manifold in $c_{0}^{*}$ is dense in $c_{0}^{*}$, it follows that $c_{0}$ has property D. Also, it follows from Theorem 3.1 that any reflexive space has property $D$. In particular then, $l_{p}$ for $1<p<\infty$ has property $\mathrm{D}$. We will now show that $l_{1}$ does also. (I do not know whether $l_{\infty}$ has property D.)

Definition 5.3. Suppose that $V=\left\{v_{1}, v_{2}, \cdots, v_{k}\right\}$ is a finite number set with only $k$ elements. The statement that the number $w$ is a proper sum of $V$ means that there is a finite sequence $q_{1}, q_{2}, \cdots$, $q_{k}$ with only $k$ terms such that each $q_{i}$ is either 1 or 2 and such that $w=\sum_{i=1}^{k}\left[\left(v_{i}\right)(-1)^{q_{i}}\right]$.

The proof of the next lemma is a straightforward induction argument and is omitted here.

Lemma 5.1. Suppose that $n$ is a positive integer such that $n-2$ is a multiple of 4. Then if $m$ is an even positive integer less than or equal to $n-2, m$ is a proper sum of the set of integers in the interval [1,n-2].

Lemma 5.2. Suppose that $V$ denotes the set to which the point $f=\left(f_{1}, f_{2}, \cdots\right)$ of $l_{\infty}$ belongs if and only if $\left|f_{n}\right|=1$ for each $n$. Then $\mathrm{L}(V)$ is dense in $l_{\infty}$.

Proof. Suppose that $x=\left(x_{1}, x_{2}, \cdots\right)$ is a point of $l_{\infty}$. Let $[a, b]$ denote a number interval containing the range of $x$. Suppose that $h$ is a positive number. Let $n$ be a positive integer such that $n-2$ is a multiple of 4 and such that the set $\{a, a+2(b-a) / n, a+4(b-a) / n$, $\cdots, b\}$ partitions $[a, b]$ into subintervals of length less than $h / 2$. Then for each term $x_{i}$ of $x$, there is an even positive integer $m\left(x_{i}\right) \leqq n-2$ such that $\left|x_{i}-\left[a+m\left(x_{i}\right)(b-a) / n\right]\right|<h / 2$. By Lemma $5.1, m\left(x_{i}\right)$ is a proper sum of $1,2, \cdots, n-2$, say $m\left(x_{i}\right)$ $=\sum_{k=1}^{n-2}\left[(k)(-1)^{q_{k}\left(x_{i}\right)}\right]$. Let $F_{0}$ denote the point of $l_{\infty}$ each of whose terms is 1 and for $j=1,2, \cdots, n-2$ let $F_{j}$ denote the point of $l_{\infty}$ whose $k$ th term is $(-1)^{\left(j q x_{k}\right)}$. Then the point

$$
F=a F_{0}+\sum_{j=1}^{n-2}\left[(j(b-a) / n) F_{j}\right]
$$

is in $\mathrm{L}(V)$ and $\|x-F\|<h$. Thus $\mathrm{L}(V)$ is dense in $l_{\infty}$.

Theorem 5.2. The space $l_{1}$ has property D.

Proof. Identify the points of $l_{1}^{*}$ with the points of $l_{\infty}$ in the usual manner. Then each point $f=\left(f_{1}, f_{2}, \cdots\right)$ of the set $V$ in Lemma 5.2 is the unique extension of the smooth point $x=\left(x_{1}, x_{2}, \cdots\right)$ of $l_{1}$ such that $x_{i}=1 / 2^{i}$ if $f_{i}=1$ and $x_{i}=-1 / 2^{i}$ if $f_{i}=-1$. Since $\mathrm{L}(V)$ is dense in $l_{\infty}$ it follows from Theorem 5.1 that $l_{1}$ has property D. 
By arguments similar to the preceding ones, the space $c$ of convergent number sequences can be shown to have property $\mathrm{D}$. The space $c^{*}$ has property D since it is congruent with $l_{1}$.

While obtaining the preceding theorems, I had conjectured that every Banach space had property $D$. That this is not the case was pointed out to me by Professor W. P. Coleman, who proved that the space $C$ of continuous real valued functions from $[0,1]$ failed to have property $\mathrm{D}$ when given the norm $\|P\|=\max _{t \in[0,1]}|P(t)|$. The construction of his counterexample may be outlined as follows: for each number $x$ in $[0,1]$ let $f_{x}$ denote the point of $C^{*}$ such that $f_{x}(P)=P(x)$ for each $P$ in $C$; let $R=\left\{f_{x} \mid x\right.$ in $\left.[0,1]\right\}$ and let $K=\mathrm{L}(R)$. Then $K$ is a $d$-manifold in $C^{*}$, but the point $g$ of $C^{*}$ such that $g(P)=\int_{0}^{1} P d I$ for each $P$ in $C$ is not in the closure of $K$.

M. M. Day proved [2] that every separable Banach space is isomorphic to a space which is strictly convex and smooth. Then every separable Banach space (and hence $C$ ) is isomorphic to a space with property $D$, and property $D$ is not preserved under isomorphisms. It would be nice to know whether every Banach space is isomorphic to a space with property $D$.

\section{REFERENCES}

1. E. Bishop and R. Phelps, A proof that every Banach space is subreflexive, Bull. Amer. Math. Soc. 67 (1961), 97-98.

2. M. M. Day, Strict convexity and smoothness of normed spaces, Trans. Amer. Math. Soc. 78 (1955), 516-528.

3. J. Dixmier, Sur une thêorème de Banach, Duke Math J. 15 (1948), 1057-1071.

4. A. F. Ruston, Conjugate Banach spaces, Proc. Cambridge Philos. Soc. 53 (1957), 576-580.

5. A. Wilansky, Functional analysis, Blaisdell, New York, 1964.

Auburn University 